\title{
Iatrogenic Tension Pneumothorax after Surgical Tracheostomy in a Child with Idiopathic Subglottic Stenosis - case report
}

\author{
Sang Yoong Park, Woo jae Yim, Joon Ho Jeong, Jeongho Kim, Seung-Cheol Lee, So Ron Choi, Jong-Hwan Lee, \\ Chan Jong Chung
}

Department of Anesthesiology and Pain Medicine, Dong-A University College of Medicine, Busan, Korea

Tracheostomy is increasingly performed in children for upper airway anomalies. Here, an 18-month-old child (height $84.1 \mathrm{~cm}$, weight $12.5 \mathrm{~kg}$ ) presented to the emergency department with dyspnea, stridor, and chest retraction. However, exploration of the airways using a bronchoscope failed due to subglottic stenosis. Therefore, a surgical tracheostomy was successfully performed with manual mask ventilation. However, pneumomediastinum was found in the postoperative chest radiograph. Although an oxygen saturation of $99 \%$ was initially maintained, oxygen saturation levels dropped, due to sudden dyspnea, after 3 hours. A chest radiograph taken at this time revealed a left tension pneumothorax and small right pneumothorax. Despite a needle thoracostomy, the pneumothorax was aggravated, and cardiac arrest occurred. Cardiopulmonary-cerebral resuscitation was performed, but the patient was declared dead 30 minutes later. This study highlights the fatal complications that can occur in children during tracheostomy. Therefore, close monitoring, immediate suspicion, recognition, and aggressive management may avoid fatal outcomes.

Key Words: Pediatrics, Pneumomediastinum, Tension pneumothorax, Tracheostomy, Thoracostomy

Tracheostomy is increasingly being performed in children, leading to improvements in neonatal and pediatric ICU care; it is frequently performed in children with upper-airway anomalies. However, there are several complications associated with tracheostomy. Some early complications include air leaks, injury to surrounding tissues, pulmonary edema, respiratory arrest, airway obstruction, and injury caused by tube placement. Delayed complications include airway obstruction, tracheoesophageal fistula, and swallowing problems, some of which can be life-threatening in children. ${ }^{1}$ Here, we report a case of a fatal bilateral pneumothorax complication with surgical tracheostomy in an 18month-old child.

\section{CASE}

An 18-month-old child (height $84.1 \mathrm{~cm}$, weight $12.5 \mathrm{~kg}$ ) presented to the emergency department

Corresponding Author: Jeongho Kim, Department of Anesthesiology and Pain Medicine, Dong-A University College of Medicine, 26, Daesingongwon-ro, Seo-gu, Busan 49201, Korea Tel: +82-51-240-5390 Fax: +82-51-247-7819 E-mail: surfy07@google.com 
with dyspnea, stridor, and chest retraction. Symptoms aggravated despite treatment with a nebulizer and steroid. Computed tomography was performed after admission where no foreign bodies were found. A flexible bronchoscope was used for exploration of the airways by the pediatric resident. However, the presence of subglottic stenosis made advancing the scope into the trachea, via the vocal cord, difficult (Fig. 1). The severity of the patient's dyspnea increased. Therefore, we decided to use a rigid ventilating bronchoscope for exploration and to perform tracheostomy with controlled ventilation under general anesthesia.

When the patient was admitted to an operation room, initial vital signs showed a blood pressure of $115 / 72 \mathrm{~mm} \mathrm{Hg}$, a heart rate of 114 beats/min, a respiratory rate of $32 / \mathrm{min}$, and an oxygen saturation of $92 \%$. After induction of anesthesia with $25 \mathrm{mg}$ of ketamine and $5 \mathrm{mg}$ of rocuronium, the otolaryngologist attempted to insert a rigid bronchoscope (Karl Storz, Tuttlingen, Germany) with an outer diameter of $4.0 \mathrm{~mm}$. However, insertion failed beyond the subglottic stenosis. The patient displayed a Cormack-Lehane classification grade of 1, and intubation was attempted using a wire-reinforced endotracheal tube (Mallinckrodt ReinforcedTM, Covidien, Dublin, Ireland) with an inner diameter of 3.5 and 4.0 $\mathrm{mm}$. However, the endotracheal tube failed to pass the vocal cord. We did not make an additional attempt due to the surrounding tissue edema and risk of complete airway obstruction. We therefore decided to perform a tracheostomy with manual mask ventilation for the management of dyspnea. Anesthesia was maintained with inhalational sevoflurane. Surgical tracheostomy was performed with manual mask

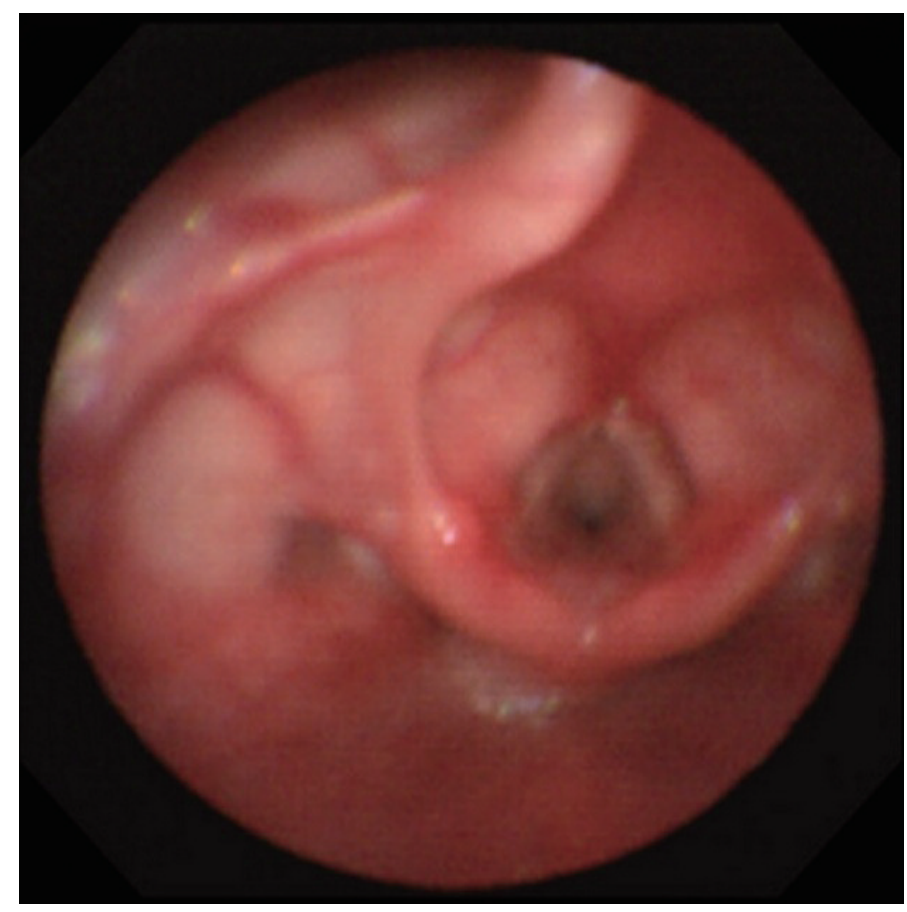

Fig. 1. Bronchoscopic view of subglottic stenosis 
ventilation at set adjustable pressure-limiting pressure levels of $10 \mathrm{cmH} 2 \mathrm{O}$, and a $4.5-\mathrm{mm}$ tracheostomy tube was inserted by an otolaryngologist. The duration of the surgery was 55 minutes, and no hypoxic events occurred during anesthesia.

At the post-anesthesia care unit following surgery, oxygen was supplied via a T-piece at about $2 \mathrm{~L} / \mathrm{min}$ and an oxygen saturation of $100 \%$ was maintained. The patient was spontaneously breathing, with improved symptoms of dyspnea. However, a pneumomediastinum was found in the postoperative chest radiograph (Fig. 2). At the ward, an oxygen saturation of $99 \%$ was maintained with oxygen supply via T-piece about 2 $\mathrm{L} / \mathrm{min}$ for 3 hours. However, sudden dyspnea occurred and oxygen saturation levels dropped to $88 \%$. After manual ventilation, oxygen saturation rose to $99 \%$ temporarily, dyspnea occurred once again and oxygen saturation dropped to $92 \%$. A blood clot was found in the tracheostomy tube. Thus, the clot was removed. However, suction catheter insertion was not easy even after removing the clot, and the tracheostomy tube was changed. The $4.5-\mathrm{mm}$ tracheostomy tube was removed due to concerns regarding tracheal wall narrowing, and a 4.0-mm tracheostomy tube was inserted by an otolaryngology resident. A chest radiograph taken at this time revealed a left tension pneumothorax and small right pneumothorax (Fig. 3). Needle thoracostomy was performed by a pediatric resident using a 20 -gauge angiocatheter needle, and $200 \mathrm{cc}$ of air was aspirated. The following chest radiograph showed the existing left pneumothorax, but an aggravated right pneumothorax (Fig. 4). During tube thoracostomy, which was performed by a thoracic surgeon, the pulse rate decreased to 70 beats/min,
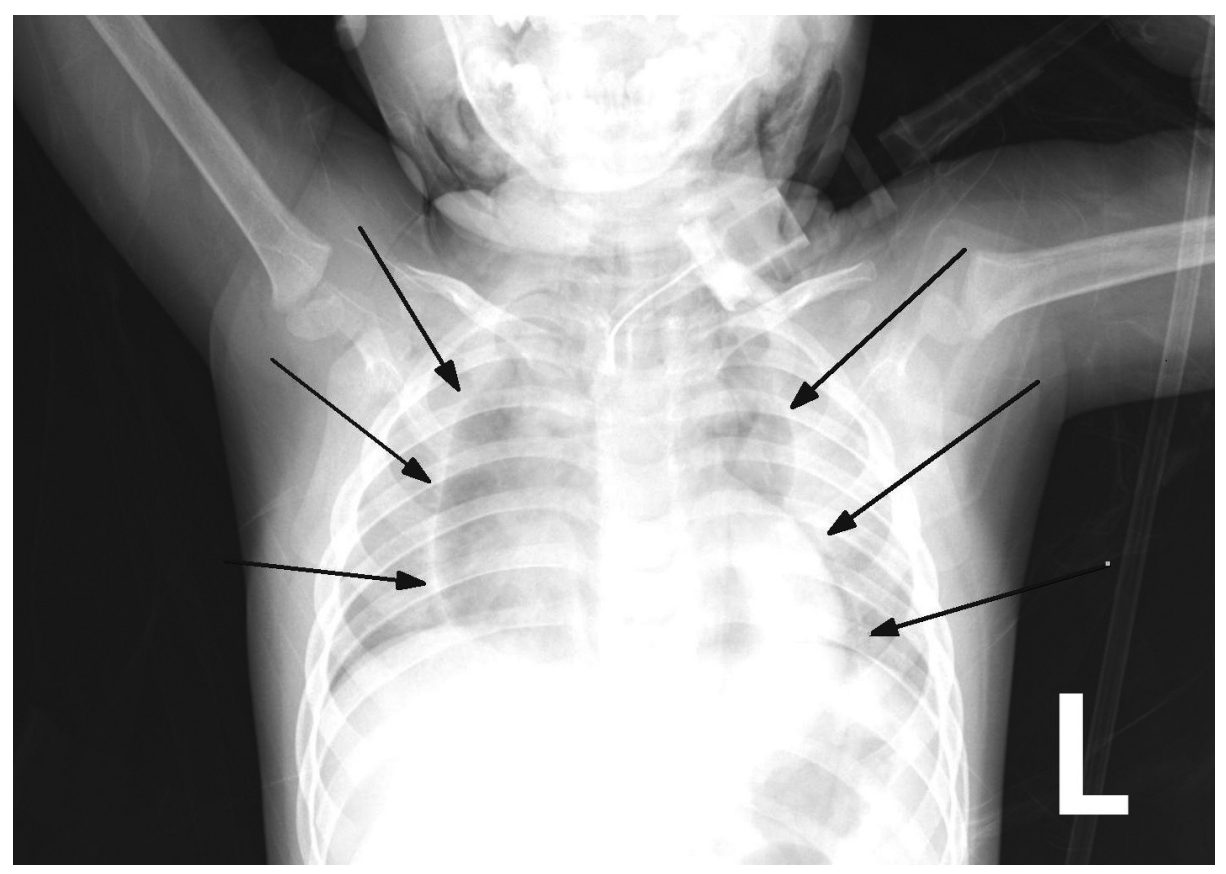

Fig. 2. A chest radiograph showing the pneumomediastinum(arrows) after tracheostomy 


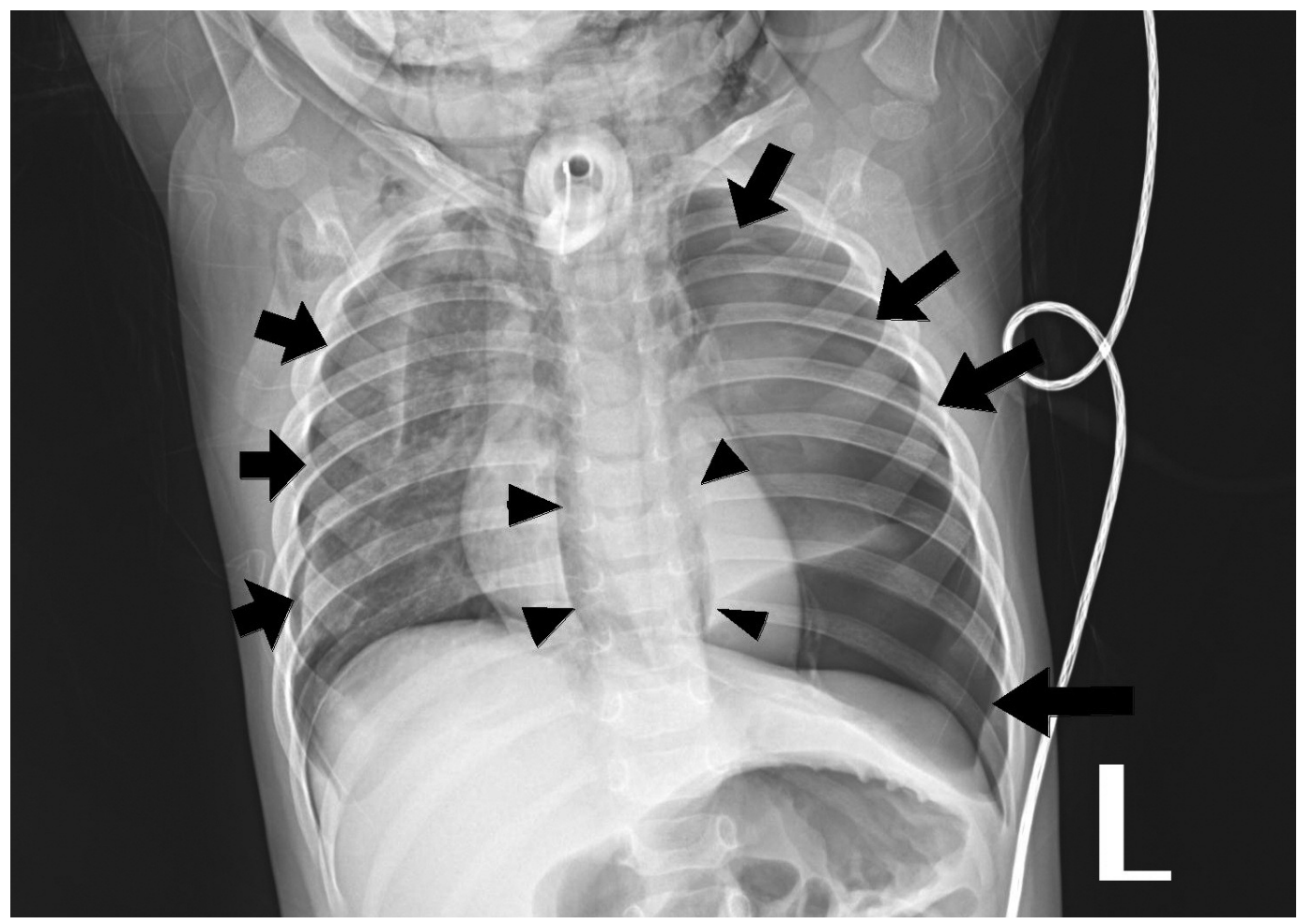

Fig. 3. A chest radiograph showing the pneumomediastinum(arrow heads), the right pneumothorax and left tension pneumothorax(black arrows)

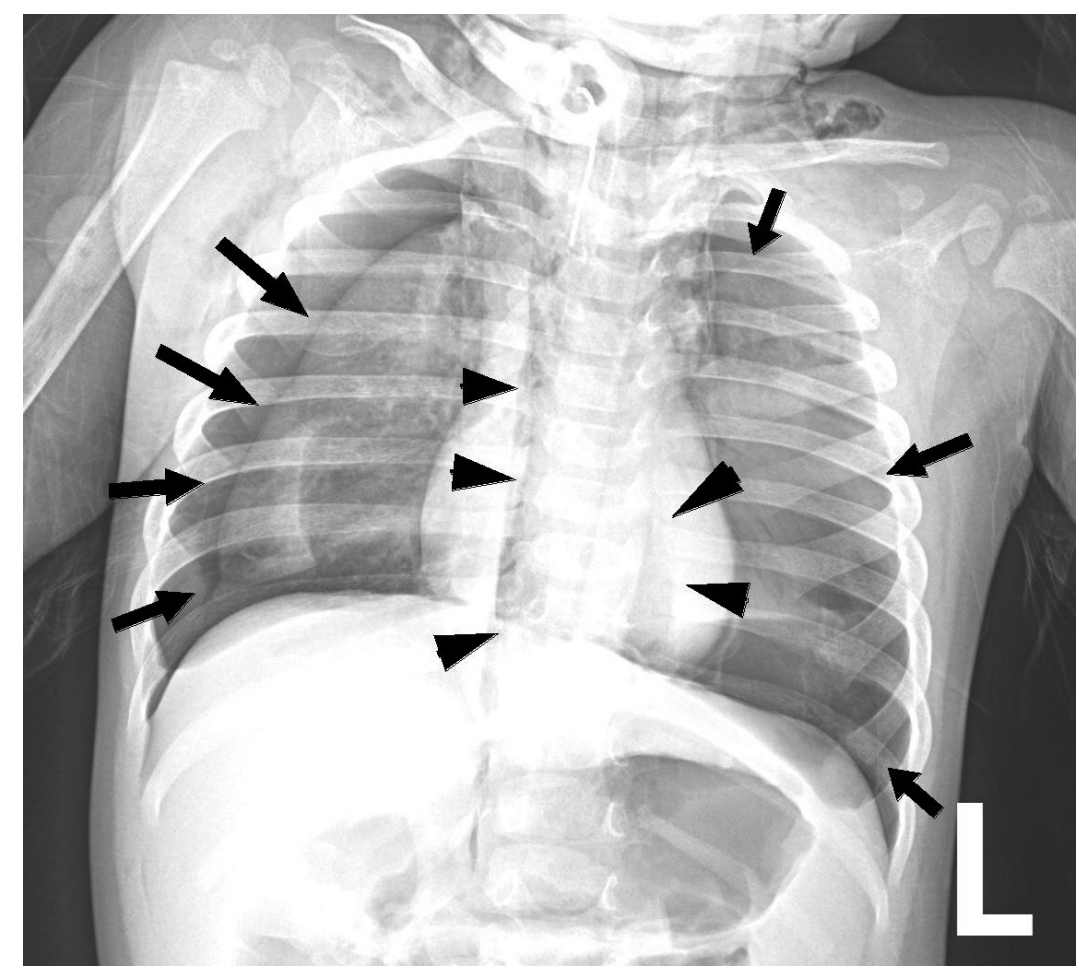

Fig. 4. A chest radiograph showing the pneumomediastinum(arrow heads) and the bilateral pneumothorax (black arrows) 
with an oxygen desaturation of up to $45 \sim 55 \%$. CPCR was performed. Epinephrine $120 \mathrm{mcg}$ was injected every $5 \mathrm{~min}$, and $10 \mathrm{mEq}$ of sodium bicarbonate was injected thrice. But the patient was declared dead 30 minutes later.

\section{DISCUSSION}

Many complications can arise from tracheostomy being performed in children. For example, pneumomediastinum can occur in children who have undergone tracheostomy, through dissection of air between the deep and superficial cervical fascia. In order to prevent pneumomediastinum, minimizing the pretracheal and paratracheal dissection is essential. ${ }^{1}$ In the absence of associated comorbidities, spontaneous pneumomediastinum can be managed with observation without the need for further imaging. ${ }^{2}$ In our case, we failed to consider the possibility of tracheal injury despite the presence of pneumomediastinum in the postoperative chest radiography. Therefore, we overlooked the fact that the pneumomediastinum could be worsened by continuous manual ventilation. Pneumothorax most commonly occurs due to an increase in the pressure gradient between the mediastinum and pleural space. Presence of pneumomediastinum allows gas to rupture the mediastinal pleura, causing it to enter the pleural space. ${ }^{3}$ Air from the mediastinum can migrate to the pericardium and cause pneumothorax and subcutaneous emphysema. ${ }^{4}$ The most efficient mechanism that has been described to prevent the risk of developing pneumothorax involves maintaining the peak inspiratory pressures low. ${ }^{5}$ In this situation, a fiberoptic bronchoscopic assessment is recommended to identify the site and severity of the injury. While a small tracheal injury can be managed conservatively, a larger tear of over $1 \mathrm{~cm}$ requires surgical repair. ${ }^{6}$ Additionally, using the fiberoptic bronchoscope as a guide, prevents the tracheostomy catheter being inserted into a false cavity. This procedure can also prevent tracheal wall injury caused by the tracheostomy tube. ${ }^{7}$ Therefore, tracheostomy guided by a fiberoptic bronchoscope is recommended in pediatric patients.

In our case, the pneumothorax was thought to develop from pneumomediastinum, and was exacerbated by manual ventilation after desaturation. Moreover, the blood clot in the tracheostomy tube could have cause airway obstruction and aggravated the pneumothorax. Tension pneumothorax occurs when a one-way valve is created between the lung and pleura. Air accumulates in the pleural cavity with every breath, and intrapleural pressure is elevated. This leads to a shrinkage in the ipsilateral lung, and the mediastinum is pushed to the opposite side. This reduces venous return, resulting in a cardiac arrest. During tension pneumothorax, chest radiological findings show a hemi-diapragmatic depression, increased rib separation, increased thoracic volume, ipsilateral flattening at the heart border, and a contralateral mediastinal deviation. ${ }^{1,8,9}$ In our case, tension pneumothorax, where the ipsilateral hemidiaphragm is pushed down was not shown in the initial postoperative chest radiograph, but 
instead was found later (Fig. 2, 3). In such case, a prompt decompression is crucial wherein a needle thoracostomy followed by a tube thoracostomy must be performed. The site for needle thoracostomy is generally the midclavicular line of the second rib in the anterior chest. Recently, the American College of Surgeons has recommended use of the fourth or fifth intercostal space at the anterior axillary line, at the same location used for a tube thoracostomy. ${ }^{10}$ However, several problems have been identified regarding needle thoracostomy, such as inappropriate length of the needle, its inability to puncture through the chest wall, catheter being tangled, and drainage failure. Needle thoracostomy needs to be repeated before adequate decompression is confirmed and a chest tube is inserted. ${ }^{8,11}$ In our case, after the needle thoracostomy, decompression was not enough in the left pneumothorax. Moreover, the right pneumothorax worsened due to manual ventilation. Finally, a cardiopulmonary collapse occurred, and the patient showed hypotension, bradycardia, and desaturation. Despite CPCR, the patient was declared dead after 30 minutes.

In conclusion, fatal complications can occur in children during tracheostomy. Complications such as posterior tracheal wall injury, pneumomediastinum, pneumothorax, and cardiac arrest can develop. Pneumomediastinum, particularly developed after tracheostomy, needs to be monitored closely and treated aggressively. While performing a tracheostomy, regardless of whether it is a surgical tracheostomy or percutaneous dilatational tracheostomy, use of a fiberop- tic bronchoscope can prevent life-threatening complications like tracheal wall injury. Inadvertent manual ventilation can also aggravate the situation, especially in a child. Therefore, immediate suspicion of potential complications and aggressive management, such as thoracostomy, can reduce fatal outcomes.

\section{REFERENCES}

1. Watters KF. Tracheostomy in infants and children. Respir Care 2017;62:799-825.

2. Fitzwater JW, Silva NN, Knight CG, Malvezzi L, Ramos-Irizarry C, Burnweit CA. Management of spontaneous pneumomediastinum in children. J Pediatr Surg 2015;50:983-6.

3. De la Matta-Martín M, Galán MJ, Gallego J. Pneumomediastinum and pneumothorax during emergency tracheotomy under spontaneous ventilation: Macklin meets Müeller? Rev Esp Anestesiol Reanim 2016;63:231-4.

4. Gasser CR, Pellaton R, Rochat CP. Pediatric Spontaneous Pneumomediastinum:Narrative Literature Review. Pediatr Emerg Care 2017;33:370-4.

5. EI-Nawawy AA, AI-Halawany AS, Antonios MA, Newegy RG. Prevalence and risk factors of pneumothorax among patients admitted to a pediatric intensive care unit. Indian J Crit Care Med 2016;20:453-8.

6. Gupta P, Modrykamien A. Fatal case of tension pneumothorax and subcutaneous emphysema after open surgical tracheostomy. J 
Intensive Care Med 2014;29:298-301.

7. Shen G, Yin H, Cao Y, Zhang M, Wu J, Jiang $\mathrm{X}$, et al. Percutaneous dilatational tracheostomy versus fibre optic bronchoscopyguided percutaneous dilatational tracheostomy in critically ill patients: a randomized controlled trial. Ir J Med Sci 2019;188:675-81.

8. Leigh-Smith S, Harris T. Tension pneumothorax - time for a re-think? Emerg Med J 2005;22:8-16.

9. Teplick SK, Clark RE. Various faces of ten- sion pneumothorax. Postgrad Med 1974;56:8792.

10. Laan DV, Vu TD, Thiels CA, Pandian TK, Schiller HJ, Murad MH, et al. Chest wall thickness and decompression failure: A systematic review and meta-analysis comparing anatomic locations in needle thoracostomy. Injury 2016;47:797-804.

11. Jones R, Hollingsworth J. Tension pneumothoraces not responding to needle thoracentesis. Emerg Med J 2002;19:176-7. 